BMJ Open Sport \& Exercise Medicine

\title{
Can resistance training improve the symptoms of polycystic ovary syndrome?
}

\author{
Paraskevi Pericleous, Savvas Stephanides
}

To cite: Pericleous $\mathrm{P}$, Stephanides S. Can resistance training improve the symptoms of polycystic ovary syndrome? BMJ Open Sport \& Exercise Medicine 2018;4:e000372. doi:10.1136/ bmjsem-2018-000372

- Additional material is published online only. To view please visit the journal online (http://dx.doi.org/10.1136/ bmjsem-2018-000372).

Accepted 17 July 2018
Check for updates

\section{(c) Author(s) (or their} employer(s)) 2018. Re-use permitted under CC BY-NC. No commercial re-use. See rights and permissions. Published by BMJ.

1

Health eResearch Center, School of Health Sciences, Faculty of Biology, Medicine and Health, The University of Manchester, Manchester, UK

Correspondence to Dr Paraskevi Pericleous; paraskevi.pericleous@ manchester.ac.uk

\section{ABSTRACT}

Objectives It has been suggested that the symptoms of Polycystic Ovary Syndrome (PCOS) could be improved by resistance training.

Design This review focuses on identifying studies that use resistance training to examine how it can affect the symptoms of polycystic ovary syndrome (PCOS).

Methods Medline, Cochrane, Dare and PubMed databases were searched to find relevant articles. All studies were assessed in terms of their design and methods.

Results We found 10 studies that used resistance training to examine how it affects the symptoms of PCOS. Conclusions There is a need for further investigation. Most studies that examine the effects of resistance training on the symptoms of PCOS needed to consider the diet (macronutrient) of the participants along with the resistance training to have a better picture of how resistance training can affect the PCOS symptoms. Many questions are still unanswered.

\section{INTRODUCTION}

Polycystic ovary syndrome (PCOS) affects the way ovaries work by causing a dysfunction, ${ }^{1}$ and it is considered a genetic disease. ${ }^{2}$ It is believed that $7 \%-15 \%$ of premenopausal women are affected worldwide (this depends on the diagnostic criteria). ${ }^{2}$ Specifically, the prevalence of PCOS according to the Rotterdam criteria is $10 \%(95 \%$ CI $7 \%$ to $13 \%) .{ }^{3}$ According to other diagnostic criteria such as the National Institutes of Health (NIH) and the Androgen Excess and PCOS Society, the prevalence is $6 \%(95 \%$ CI $5 \%$ to $8 \%$ ) and $10 \%(95 \%$ CI $7 \%$ to $13 \%)$, respectively. ${ }^{3}$ Genes and the environment are partly responsible for PCOS. Sedentary lifestyle, bad eating habits and obesity can worsen the adverse effects of PCOS. ${ }^{4}$

In April 1990, a conference was sponsored by the NIH where it was decided that the syndrome includes (1) hyperandrogenism and/or hyperadrogenaemia, (2) oligo-ovulation and (3) exclusion of known disorders. ${ }^{5}$ In May 2003, in a conference in Rotterdam, these changed, where (3) was excluded and
What is already known?

Studies have been investigating the effects of weight training on the symptoms of polycystic ovary syndrome (PCOS).

\section{What are the new findings?}

- Diet and specific macronutrient breakdown is an important factor that has often been neglected by studies.

- We need more studies that consider diet and specific macronutrient breakdown along with weight training to assess the best way to improve the PCOS symptoms.

replaced. According to the Rotterdam criteria, two of the following need to be present: (1) oligo-ovulation or anovulation, (2) clinical and/or biochemical signs of hyperandrogenism, or (3) polycystic ovaries..$^{5}$ So, after May 2003, two more categories appeared: ovulatory women with polycystic ovaries and hyperandrogenism, and oligo-ovulatory women with polycystic ovaries but without hyperandrogenism. ${ }^{5}$ It seems, however, that many experts consider the Rotterdam 2003 criteria for diagnosing PCOS as premature or obsolete and that there is an urgent need to update these again. ${ }^{5}$

Some of the symptoms of PCOS include menstrual disturbance, obesity, hirsutism, androgenic alopecia, acne and acanthosis nigricans, ${ }^{6} \quad$ oligo-ovulation/anovulation and oligomenorrhoea/amenorrhoea which contribute to infertility and increased risk of miscarriages, ${ }^{78}$ mental health problems, thinning scalp hair, and ovarian cysts. ${ }^{9}$ PCOS is associated with obesity, cardiovascular disease (and hypertension), type II diabetes and metabolic syndrome. ${ }^{10}{ }^{11}$ It can be also linked with breast ${ }^{12}$ and ovarian cancer. ${ }^{13}$ However, not all studies agree. There seems to be contradictory evidence for both ovarian and breast cancer. $^{14}$ 
PCOS is a disease commonly found in women. Because of the aforementioned symptoms of PCOS, which have been characterised as 'the thief of womanhood' by Kitzinger and Willmott, ${ }^{15}$ and all the possible associations with other diseases, there is an urgent need to treat these symptoms.

Women with PCOS have insulin resistance that is independent of body mass index (BMI) ${ }^{16}$ Postbinding defect in receptor signalling that disturbs the metabolic pathways in classic insulin target tissues, constitutive activation of serine kinases in the MAPK-ERK pathway and androgen levels contribute to this insulin resistance. ${ }^{17}$ In layman's terms, genetics and lifestyle affect hormonal changes, insulin resistance and androgen increase, and both can affect the ovarian follicles, may cause anovulation and increase oestrogen levels, which then cause menstrual disturbances and subfertility. Also, insulin resistance can cause diabetes and metabolic syndrome, which then increase cardiovascular risk. In addition to these, increase in androgen levels can cause hirsutism and acne. All these then create psychosocial issues related to body image, self-esteem, depression and anxiety. ${ }^{18}$

It has been shown that lifestyle-related metabolic diseases, such as being overweight or obese, and insulin resistance cause exacerbation of $\operatorname{PCOS}^{19} 20$ (insulin resistance through its effect on androgen production ${ }^{21}$ ). These two metabolic diseases in PCOS may be influenced by sedentary behaviour and excess calorific intake. ${ }^{22}$

The PCOS Australian Alliance published exercise prescription guidelines that included exercising at least 150 min per week, 90 min of which should be an aerobic activity of moderate to high intensity $(60 \%-90 \%$ of maximum heart rate) to optimise clinical outcomes. ${ }^{18}$ There is no mention of resistance training to manage PCOS. ${ }^{18}$ However, there is a physiological rationale to prescribe resistance training to patients with PCOS. Insulin resistance causes exacerbation of PCOS and resistance training has been shown to improve insulin resistance. $^{21}$

Resistance training (also called weight or strength training) is the type of exercise that requires the body (muscles) to push against force that is practised against it. It includes bodyweight exercises, plyometrics, use of resistance bands, free weights or machine equipment. ${ }^{23}$

Resistance training benefits the musculoskeletal system, ${ }^{21}$ and prevents osteoporosis, lower back pain and so on. It also improves insulin resistance, glucose metabolism and resting metabolic rate, and lowers body fat. ${ }^{24}$ Furthermore, it can increase insulin sensitivity in type II diabetes. However, resistance training is not currently recommended to patients with PCOS, ${ }^{21}$ despite the effect that it can have on the PCOS symptoms. Some authors recommend resistance training 2-3 non-consecutive days per week, at $60 \%$ to $70 \%-85 \%$ of one-repetition maximum (1RM), targeting all major muscle groups, and progress according to tolerance. ${ }^{21}$

There are numerous studies that have examined the association of diet and exercise as an intervention for the clinical outcomes associated with PCOS. It has been found that such an intervention is crucial to improving body composition and cardiorespiratory fitness in patients with PCOS. ${ }^{25}$ It improves the levels of Follicle-stimulating hormone (FSH), sex hormone-binding globulin (SHBG), total testosterone, androstenedione, and the Free Androgen Index (FAI) and Ferriman-Gallwey (FG) score. ${ }^{25}{ }^{26}$ It has been found that exercise as a therapy can improve ovulation, insulin resistance and weight loss, ${ }^{27}$ and that women with PCOS are more likely to be using weight management practices than the healthy population. $^{28}$

We believe it is more informative to combine resistance training with the appropriate dieting routine/habits to achieve specific goals. A systematic review found that weight loss can improve PCOS symptoms independent of the dietary composition. ${ }^{29}$ However, other studies have shown that different macronutrient intakes may lead to different results even when other factors remain unchanged, and that the effects of resistance training can be optimised with the appropriate diet habits. ${ }^{30}$ Three different groups-resistance training, diet, and resistance training with diet-were compared, and even though all of them had significant fat mass (FM) reductions only the resistance training group had an increase in lean mass. ${ }^{31}$ Also, when resistance-trained athletes restrict calories or have a low body fat, their protein needs increase. ${ }^{32}$ However, a study on hypercholesterolaemic obese women found that even though resistance training improved low-density lipoprotein cholesterol (LDL-C) and total cholesterol, a diet lower in protein intake had a greater reduction in LDL-C (all groups had weight reduction). ${ }^{33}$ Obese women on a carbohydrate-restricted diet and are resistance-trained had greater weight loss, fat loss and more favourable changes in health markers compared with the ones with a higher carbohydrate diet, ${ }^{34}$ and greater reductions in blood glucose were achieved with a high-protein diet in women with higher insulin resistance. ${ }^{34}$ Macronutrient intake can possibly influence the circulating levels of insulin in obese women following a regular exercise programme that includes resistance training. ${ }^{35}$ A study concluded that ketogenic diet with resistance training can reduce body fat without changing lean body mass significantly; when the ketogenic diet is switched to a regular diet, there is an increase in lean body mass without changing the FM significantly. ${ }^{36}$ Thus, we believe that examining the effects of resistance training on the PCOS symptoms needs to be accompanied and regulated with calorific and specific macronutrient dietary habits to understand the mechanisms that can mostly assist in improving those symptoms.

\section{METHODS}

We have searched Medline, Cochrane, Dare and PubMed databases in February 2017 and then again in February 2018 to find relevant articles, and then additional ones from these and from the bibliography that we already 
had. We used 3 terms for PCOS (PCOS OR polycystic ovary syndrome OR polycystic ovaries syndrome) and 10 terms for resistance training (weight-training OR weight training OR weight-lifting OR weight lifting OR lifting OR resistance training OR resistance-training OR weights OR exercise OR exercise therapy). All studies are discussed. We find possible limitations and how these can be improved.

\section{RESULTS}

We have included any study that mentioned that participants were women with PCOS and were involved in any sort of resistance training. We have found 10 studies (6 of these studies are actually 2 different ones with different outcomes) that were relevant to what we were looking for, dating from 2008 to 2017. Four of them were in Australia, three in Brazil and one in the USA (pilot study), and five of them are randomised controlled trials. All, but one, use the Rotterdam diagnostic criteria.

\section{DISCUSSION}

Study sizes vary from 12 and 15 (pilot studies) ${ }^{37} 38$ to $122,{ }^{39}$ and the study duration goes from 12 weeks ${ }^{37}$ to open-end, but allowed up to 14 weeks $^{40}$ to 4 months. ${ }^{41} 42$ The inclusion criteria were quite consistent as most of the studies included at least overweight women with PCOS who were at least 18 years old. The exclusion criteria for all studies were pregnancy (all studies except one that encouraged them to continue ${ }^{39}$ ), hormonal contraceptive use and fertility treatments. Three studies excluded participants due to breast feeding, cardiovascular history, liver, kidney or respiratory disease, diabetes, uncontrolled hypertension, malignancy, reproductive disorders unrelated to PCOS, thyroid abnormalities, and non-classical adrenal hyperplasia. ${ }^{43-45}$ Four studies excluded participants who were playing sports or were exercising prior to the study. ${ }^{41} 43-45$

All studies had dropouts, the most common reason being pregnancy (except one, ref ${ }^{39}$ ). Other reasons included loss of contact, work commitments, illness/ injury, moving, time restraints and unable to comply with the requirements of the study. There is only one study that did not mention any reasons for dropout. ${ }^{40}$ The main patient characteristics in all studies included age, BMI and weight. Some studies included body FM, abdominal FM or body fat percentage,${ }^{42} 45$ and others included waist circumference or waist to hip ratio. ${ }^{39} 41$

Contrary to our expectation, not all studies have described in detail what their intervention included. ${ }^{39}$ They define 'lifestyle intervention' as a calorie-controlled diet from healthy food options and at least $150 \mathrm{~min}$ of exercise (90 min aerobic) per week. Two trained coaches (nutritionist and exercise physiologist) introduced diet and exercise (aerobic and progressive resistance). This description is missing important information on participants' training and diet, and we cannot draw any conclusions about it.
Other studies used three groups. One was diet and aerobic (DA), the other was diet and aerobic resistance (DC), and the last was diet only (DO) ${ }^{43-45}$ The exercises were supervised, and the participants completed a weekly exercise diary and a daily food quantity checklist. The aerobic exercise consisted of walking/jogging five times a week: $25-30 \mathrm{~min}$ at $60 \%-65 \%$ maximum heart rate during the first week to $45 \mathrm{~min} 75 \%-80 \%$ maximum heart rate by the end of the study. The resistance training consisted of five resistance exercises (twice a week on non-consecutive days): bench press, lat pulldown, leg press, knee extension and sit-ups. The training load for the first 2 weeks was $50 \%-60 \%$ of $1 \mathrm{RM}$ and increased to $65 \%-75 \%$ of 1RM the following weeks. Load was increased if the participants could perform 3 sets of 12 repetitions with that load. In terms of diet, participants had energy-restricted, high-protein diet for a planned weight loss of 8-12 kg over the study period, and the macronutrients comprise $30 \%$ protein, $40 \%$ carbohydrates and $30 \%$ fat ( $<8 \%$ saturated). These are among the most informative studies that we have found. However, we feel that they could have added whether fibre was part of those carbohydrates or how much fibre the participants were eating and what kind of carbohydrates they were eating (high glycaemic or low). We would like to see the reason behind the choice of this macronutrient breakdown. We also feel that more priority was given to aerobic exercise and that resistance training of two times a week was probably not enough, and this could be the reason why there are no differences between the two exercise groups, with only one exception. ${ }^{38}$ When it comes to sit-ups, even though it is an important addition to any strength training regimen, we believe that when it comes to this kind of study, a more compound one should have been chosen, such as squats. One could argue though that the weight of the participants could be a barrier to doing this kind of exercises or that it is a difficult exercise for beginners. Jogging is probably not suitable for these participants either due to their weight. We believe that supervision of exercises is crucial to participants' safety and the results of the study, and supervising them was the right decision. There is also a lack of sample size and power calculation, probably due to the fact that these were the first studies on this topic.

Another study, however, did not take it as far. ${ }^{37}$ They used motivational interview (MI) with six different themes of change talk (desire, ability, reasons, need, commitment and taking steps). At individualised counselling sessions, patients were advised on food preparations, shopping tips, menu preparation, eating food with lower glycaemic index, healthy eating, calorific deficit, food categories, portions, serving sizes and minimising saturated fat. They were also advised to engage to low-impact physical activity 3-5 days/week for 30-60 min at least and resistance training 2-3 times a week engaging all major muscle groups, and walking longer distances, taking the stairs and so on. Besides the information and MI given to the participants, this study did not consist of any more action to ensure that participants followed their guidance. 
There was no diet or exercise supervision. Thus, there is no evidence that the participants actually did what they were asked to do.

There are only three studies comparing women with PCOS with healthy women. ${ }^{414} 46$ The participants first joined a protocol to learn the exercises. The study included four microcycles of 4 weeks each: 3 sets of 15 repetitions, 3 sets of 12 repetitions, 3 sets of 10 repetitions and 3 sets of 8 while doing pyramids with the load within each microcycle. They were increasing the load though on the next microcycle. They spent 1 hour per day, three times a week on progressive resistance training. Exercise intensity was increased in each microcycle and the number of repetitions was decreased, with the minimum being 8 (volume decreasing). The exercises were bench press, leg extension, front lat pulldown, leg curl, lateral raise, leg press with a $45^{\circ}$ incline, arm curl and abdominal exercise (they also did stretching). All exercises were supervised and they provided a light meal afterwards and a pair of running shoes, and funded the transportation. A major limitation to this study is that it did not mention diet at all. Any sort of training either aerobic or resistance training cannot alone affect someone's body. Diet needs to be in control also. If there was no guidance or supervision or report on their diet, then we believe we are missing some important information. For instance, the participants may think they would be on sufficient calorific deficit/surplus when in fact this would be the opposite or more/less than the amount they estimate.

We believe that these kinds of studies needed to account and monitor for specific calorific intake (and macronutrient). The ones that did not account or monitor this have probably not included some very important information. Additional details, including more limitations and the results of each study, can be found in the online supplementary appendix. From all the studies on this subject, only three of them provided dietary information. ${ }^{43-45}$

The studies concluded that all three groups had a significant bodyweight reduction. The group that included diet and aerobic (DA) and the group that included diet, aerobic and resistance training (DC) had greater reduction in FM and less reduction in fat-free mass compared with the group that was not training at all (DO). The DC group had improved their muscle strength. All groups had significant reductions in blood pressure, fasting glucose and insulin and insulin resistance, testosterone, and FAI, increase in SHBG, and improvements in their ovulations and/or menstrual cycle.

\section{CONCLUSIONS}

Contrary to our expectation, most of the studies that we found did not provide information on (or did not monitor) participants' diet. There is a need for further studies in order to understand whether resistance training can improve the PCOS symptoms. The list is not extensive, but we need to comprehend how different macronutrients with resistance training can assist symptom improvement, for example, which of high-protein, high-fat or high-carb diet is more beneficial, or whether a more balanced diet is better. We need to determine whether high glycaemic or low glycaemic carbohydrate consumption with resistance training would be a better choice for these patients. We need to consider a calorific surplus instead of a deficit for patients undergoing resistance training, whether they gain more muscle than regular women and achieve better fat loss results in the long term. In addition to this, there are studies that include aerobic or aerobic and resistance training. No study, however, used resistance training alone to examine the outcome (except one, but did not consider nutrition $^{41}$ ), and no study used compound exercises only such as squats and deadlifts. Finally, all these (exercise and nutrition) need to be under close supervision. All these and many more questions are still unanswered. There is plenty to learn and more studies to be conducted to answer these questions.

Contributors Both authors have equally identified the studies required and assessed these in terms of their design and quality.

Funding The authors have not declared a specific grant for this research from any funding agency in the public, commercial or not-for-profit sectors.

\section{Competing interests None declared.}

Patient consent Not required.

Provenance and peer review Not commissioned; externally peer reviewed.

Open access This is an Open Access article distributed in accordance with the Creative Commons Attribution Non Commercial (CC BY-NC 4.0) license, which permits others to distribute, remix, adapt, build upon this work non-commercially, and license their derivative works on different terms, provided the original work is properly cited and the use is non-commercial. See: http://creativecommons.org/ licenses/by-nc/4.0

\section{REFERENCES}

1. Rotterdam ESHRE/ASRM-Sponsored PCOS Consensus Workshop Group. "Revised 2003 consensus on diagnostic criteria and longterm health risks related to polycystic ovary syndrome". Fertil Steril 2004:81:19-25.

2. Dapas M. "Pp32-1: Identification of rare and deleterious small variants in families affected by polycystic ovary syndrome. 2016.

3. Bozdag G, Mumusoglu S, Zengin D, et al. The prevalence and phenotypic features of polycystic ovary syndrome: a systematic review and meta-analysis. Hum Reprod 2016;31:2841-55.

4. Diamanti-Kandarakis $E$, Kandarakis $H$, Legro RS. The role of genes and environment in the etiology of PCOS. Endocrine 2006;30:19-26.

5. Azziz R. Diagnosis of polycystic ovarian syndrome: The rotterdam criteria are premature. JCEM 2006;91:781-5

6. Hart R, Hickey M, Franks S. Definitions, prevalence and symptoms of polycystic ovaries and polycystic ovary syndrome. Best Pract Res Clin Obstet Gynaecol 2004;18:671-83.

7. Giudice LC. Endometrium in PCOS: Implantation and predisposition to endocrine CA. Best Pract Res Clin Endocrinol Metab 2006;20:235-44.

8. Gorry A, White DM, Franks S. Infertility in polycystic ovary syndrome: focus on low-dose gonadotropin treatment. Endocrine 2006;30:27-34.

9. National Institutes Of Health. 2012. Evidence-based methodology workshop on polycystic ovary syndrome. https://prevention.nih.gov/ docs/programs/pcos/FinalReport.pdf (accessed 15 Dec 2012).

10. Rotterdam ESHRE, P. C. O. S. ASRM-Sponsored. "Revised. consensus on diagnostic criteria and long-term health risks related to polycystic ovary syndrome.". Fertil Steril 2003;81.1:19.

11. Orio F, Muscogiuri G, Nese C, et al. Obesity, type 2 diabetes mellitus and cardiovascular disease risk: an uptodate in the management of polycystic ovary syndrome. Eur J Obstet Gynecol Reprod Biol 2016;207:214-9. 
12. Kim J, Mersereau JE, Khankari N, et al. Polycystic ovarian syndrome (PCOS), related symptoms/sequelae, and breast cancer risk in a population-based case-control study. Cancer Causes Control 2016;27:403-14.

13. Schildkraut JM, Schwingl PJ, Bastos E, et al. Epithelial ovarian cancer risk among women with polycystic ovary syndrome. Obstet Gynecol 1996;88(4 Pt 1):554-9.

14. Balen A. Polycystic ovary syndrome and cancer. Hum Reprod Update 2001;7:522-5.

15. Kitzinger $\mathrm{C}$, Willmott J. 'The thief of womanhood': women's experience of polycystic ovarian syndrome. Soc Sci Med 2002;54:349-61.

16. Cassar S, Misso ML, Hopkins WG, et al. Insulin resistance in polycystic ovary syndrome: a systematic review and meta-analysis of euglycaemic-hyperinsulinaemic clamp studies. Hum Reprod 2016;31:2619-31.

17. Diamanti-Kandarakis E, Dunaif A. Insulin resistance and the polycystic ovary syndrome revisited: an update on mechanisms and implications. Endocr Rev 2012;33:981-1030.

18. Teede HJ, Misso ML, Deeks AA, et al. Assessment and management of polycystic ovary syndrome: summary of an evidence-based guideline. Med J Aust 2011;195:S65-112.

19. Diamanti-Kandarakis $\mathrm{E}$, Christakou $\mathrm{C}$. Insulin resistance in PCOS. Farid N, Diamanti-Kandarakis E. New York: Diagnosis and management of polycystic ovary syndrome, 2009:35-61.

20. Pasquali R, Gambineri A, Pagotto U. The impact of obesity on reproduction in women with polycystic ovary syndrome. BJOG 2006;113:1148-59.

21. Cheema BS, Vizza L, Swaraj S. Progressive resistance training in polycystic ovary syndrome: can pumping iron improve clinical outcomes? Sports Med 2014;44:1197-207.

22. Moran LJ, Ranasinha S, Zoungas S, et al. The contribution of diet, physical activity and sedentary behaviour to body mass index in women with and without polycystic ovary syndrome. Hum Reprod 2013;28:2276-83.

23. Fleck SJ, Kraemer W. Designing Resistance Training Programs, 4E: Human Kinetics, 2014 Feb 14.

24. Winett RA, Carpinelli RN. Potential health-related benefits of resistance training. Prev Med 2001;33:503-13

25. Haqq L, McFarlane J, Dieberg G, et al. Effect of lifestyle intervention on the reproductive endocrine profile in women with polycystic ovarian syndrome: a systematic review and meta-analysis. Endocr Connect 2014;3:36-46.

26. Haqq L, McFarlane J, Dieberg G, et al. The effect of lifestyle intervention on body composition, glycemic control, and cardiorespiratory fitness in polycystic ovarian syndrome: $A$ systematic review and meta-analysis. Int J Sport Nutr Exerc Metab 2015;25:533-40.

27. Harrison CL, Lombard CB, Moran LJ, et al. Exercise therapy in polycystic ovary syndrome: a systematic review. Hum Reprod Update 2011;17:171-83.

28. Moran LJ, Brown WJ, McNaughton SA, et al. Weight management practices associated with PCOS and their relationships with diet and physical activity. Hum Reprod 2017;32:669-78.

29. Moran LJ, Ko H, Misso M, et al. Dietary composition in the treatment of polycystic ovary syndrome: a systematic review to inform evidence-based guidelines. J Acad Nutr Diet 2013;113:520-45.

30. Strandberg E, Edholm P, Ponsot E, et al. Influence of combined resistance training and healthy diet on muscle mass in healthy elderly women: a randomized controlled trial. $J$ Appl Physiol 2015;119:918-25.
31. Miller T, Mull S, Aragon AA, et al. Resistance training combined with diet decreases body fat while preserving lean mass independent of resting metabolic rate: A randomized trial. Int J Sport Nutr Exerc Metab 2018;28:46-54.

32. Helms ER, Zinn C, Rowlands DS, et al. A systematic review of dietary protein during caloric restriction in resistance trained lean athletes: a case for higher intakes. Int J Sport Nutr Exerc Metab 2014;24:127-38.

33. García-Unciti M, Martinez JA, Izquierdo M, et al. Effect of resistance training and hypocaloric diets with different protein content on body composition and lipid profile in hypercholesterolemic obese women. Nutr Hosp 2012;27:1511-20.

34. Kreider RB, Rasmussen C, Kerksick CM, et al. A carbohydraterestricted diet during resistance training promotes more favorable changes in body composition and markers of health in obese women with and without insulin resistance. Phys Sportsmed 2011;39:27-40.

35. Kerksick CM, Wismann-Bunn J, Fogt D, et al. Changes in weight loss, body composition and cardiovascular disease risk after altering macronutrient distributions during a regular exercise program in obese women. Nutr J 2010;9:9-59.

36. Jabekk PT, Moe IA, Meen HD, et al. Resistance training in overweight women on a ketogenic diet conserved lean body mass while reducing body fat. Nutr Metab 2010;7:17.

37. Mahoney D. Lifestyle modification intervention among infertile overweight and obese women with polycystic ovary syndrome. J Am Assoc Nurse Pract 2014;26:301-8.

38. Vizza L, Smith CA, Swaraj S, et al. The feasibility of progressive resistance training in women with polycystic ovary syndrome: a pilot randomized controlled trial. BMC Sports Sci Med Rehabil 2016;8:14.

39. Arentz S, Smith CA, Abbott J, et al. Combined lifestyle and herbal medicine in overweight women with polycystic ovary syndrome (PCOS): A randomized controlled trial. Phytother Res 2017:31:1330-40.

40. Aubuchon M, Laughbaum N, Poetker A, et al. Supervised short-term nutrition and exercise promotes weight loss in overweight and obese patients with polycystic ovary syndrome. Fertil Steril 2009;91(4 Suppl):1336-8.

41. Kogure GS, Miranda-Furtado CL, Silva RC, et al. Resistance exercise impacts lean muscle mass in women with polycystic ovary syndrome. Med Sci Sports Exerc 2016;48:589-98.

42. Miranda-Furtado CL, Ramos FK, Kogure GS, et al. A nonrandomized trial of progressive resistance training intervention in women with polycystic ovary syndrome and its implications in telomere content. Reprod Sci 2016;23:644-54.

43. Thomson RL, Buckley JD, Noakes M, et al. The effect of a hypocaloric diet with and without exercise training on body composition, cardiometabolic risk profile, and reproductive function in overweight and obese women with polycystic ovary syndrome. $J$ Clin Endocrinol Metab 2008;93:3373-80.

44. Thomson RL, Buckley JD, Lim SS, et al. Lifestyle management improves quality of life and depression in overweight and obese women with polycystic ovary syndrome. Fertil Steril 2010;94:1812-6.

45. Thomson RL, Brinkworth GD, Noakes M, et al. The effect of diet and exercise on markers of endothelial function in overweight and obese women with polycystic ovary syndrome. Hum Reprod 2012;27:2169-76.

46. Ramos FK, Lara LA, Kogure GS, et al. Quality of life in women with polycystic ovary syndrome after a program of resistance exercise training. Rev Bras Ginecol Obstet 2016;38:340-7. 